\title{
Annexin A1 is involved in resistance to 5-FU in colon cancer cells
}

\author{
HISASHI ONOZAWA, MOTONOBU SAITO, KATSUHARU SAITO, YASUYUKI KANKE, YOHEI WATANABE, \\ SUGURU HAYASE, WATARU SAKAMOTO, TERUHIDE ISHIGAME, TOMOYUKI MOMMA, \\ SHINJI OHKI and SEIICHI TAKENOSHITA
}

Department of Organ Regulatory Surgery, Fukushima Medical University School of Medicine, Fukushima 960-1295, Japan

Received June 15, 2016; Accepted October 24, 2016

DOI: $10.3892 / o r .2016 .5234$

\begin{abstract}
Resistance to 5-fluorouracil (5-FU), a key drug in the treatment of colorectal cancer, is one of the major reasons for poor patient prognosis during cancer treatment. Annexin A1 (ANXA1) is a calcium-dependent phospholipid-linked protein that is associated with drug resistance, anti-inflammatory effects, regulation of cellular differentiation, proliferation and apoptosis. Although there have been several studies investigating ANXA1 expression in drug resistant cells, the role of ANXA1 is yet to be fully understood. We therefore, in this study, generated SW480 cells resistant to 5-FU (SW480/5-FU) to evaluate ANXA1 expression. When compared to the control cells, ANXA1 expression was significantly induced in the SW480/5-FU cells. We then revealed the role of ANXA1 expression in 5-FU resistance by using overexpression and knockdown methods in colon cancer cells. Overexpression of ANXA1 induced a significant increase of cell viability to 5-FU, whereas ANXA1 knockdown induced a significant decrease of cell viability to 5-FU. Further experiments revealed that ANXA1 expression was induced by hypoxia in colon cancer cells. These results suggest that ANXA1 expression may play a critical role in 5-FU resistance and may be induced by hypoxia during cancer progression. Our results provide a possible strategy to overcome 5-FU resistance by modulating ANXA1 expression.
\end{abstract}

\section{Introduction}

Colorectal cancer is the third most common type of cancer in both females and males in the United States; however, its incidence and mortality rates have been decreasing in recent years (1). This is attributed to many factors, such as more frequent performance of surveillance colonoscopy, improvements in surgical and radiotherapy techniques, and development of new chemotherapy and molecular targeted

Correspondence to: Dr Seiichi Takenoshita, Department of Organ Regulatory Surgery, Fukushima Medical University School of Medicine, 1 Hikarigaoka, Fukushima 960-1295, Japan

E-mail: takenoss@fmu.ac.jp

Key words: colon cancer, chemoresistance, proliferation, 5-fluorouracil, Annexin A1 drugs $(2,3)$. Although chemotherapy and targeted therapy drugs have progressed over the past few decades, drug resistance is still a major problem in the treatment of colorectal cancer. Combination chemotherapy with FOLFOX (folinic acid (LV)/5-fluorouracil (5-FU)/oxaliplatin) and FORFIRI (LV/5-FU/irinotecan) provides a higher response rate and has now become the standard treatment regimen for colorectal cancer, but resistance to combination chemotherapy eventually occurs, resulting in tumor recurrence or metastasis.

Annexin A1 (ANXA1), which belongs to the Annexin superfamily, is a $37 \mathrm{kDa}$ calcium-dependent phospholipid-linked protein involved in apoptosis, anti-inflammatory effects, and the regulation of cellular differentiation and proliferation $(4,5)$. Through these functions, ANXA1 is considered to be associated with cancer development in various malignant tumors, including colorectal cancer $(6,7)$. We previously reported a case of positive ANXA1 expression by immunohistochemical (IHC) staining in breast cancer that was associated with triple-negative breast cancer $(\mathrm{P}=0.007)$ and venous invasion $(\mathrm{P}=0.028)(8)$. In vitro cell experiments revealed that ANXA1 enhanced breast cancer invasion and metastasis under hypoxia, indicating that ANXA1 was significantly associated with worse breast cancer patient outcome. We also reported a case of positive ANXA1 expression by IHC staining in colon cancer associated with venous invasion $(\mathrm{P}=0.023)$ as well as lymph node metastasis $(\mathrm{P}=0.042)(9)$. These positive cases were not statistically associated with poor survival, but appeared to be associated with worse colon cancer patient outcome.

According to recent studies, there is no doubt that drug resistance is one of the major reasons of poor patient outcome following cancer treatment. Those studies identified that ANXA1 is associated with drug resistance through modulation of the drug export mechanism of P-glycoprotein and the multidrug resistance protein (10). One of the key drugs of the FORFIRI and FOLFOX regimens is 5-FU; thus, we established a 5-FU-resistant cell line to focus on the pivotal role of ANXA1 in 5-FU resistance. The present study also implicates the 5-FU resistance by modulating ANXA1 expression in colon cancer cells.

\section{Materials and methods}

Cell culture. The colon cancer cell lines used in this study were originally obtained from the American Type Culture 
Collection (Rockville, MD, USA) and were cultured in the recommended media with $10 \%$ fetal bovine serum (FBS). These monolayer cells were maintained in a $37^{\circ} \mathrm{C}$ incubator with $5 \% \mathrm{CO}_{2}$. Cells were checked regularly under a light microscope and subcultured when they reached $80-90 \%$ confluence. For hypoxia exposure, each cell type was cultured for $24 \mathrm{~h}$ in a modulator incubator chamber (Billups-Rothenberg, Del Mar, CA, USA) at $37^{\circ} \mathrm{C}$ with $1 \% \mathrm{O}_{2}, 5 \% \mathrm{CO}_{2}$, and $94 \% \mathrm{~N}_{2}$. To mimic hypoxia, the cells were cultured for $24 \mathrm{~h}$ with $100 \mu \mathrm{M}$ cobalt chloride $\left(\mathrm{CoCl}_{2}\right)$ (Sigma-Aldrich, St. Louis, MO, USA).

IHC staining and evaluation. Colon cancer cell lines were immunostained for ANXA1 (clone 29; BD Biosciences, San Jose, CA, USA) and evaluated for staining intensity. Briefly, colon cancer cells were fixed in $10 \%$ formalin and embedded in paraffin then cut into thin $(4 \mu \mathrm{m})$ sections and stained. The expression of ANXA1 protein was evaluated by the ratio of the number of positively stained cells to negatively stained cells. Ten colon cancer cell lines were dichotomized as positive ( $\geq 5 \%$ staining) or negative ( $<5 \%$ staining) for ANXA1.

Establishment of 5-FU resistant SW480 cells. A 5-FU-resistant SW480 cell line (SW480/5-FU) was established by repeated exposure to stepwise increasing concentrations of 5-FU up to $1 \mu \mathrm{M}$, as previously described (11). The cells were cultured in RPMI-1640 medium with $10 \% \mathrm{FBS}$ in a $37^{\circ} \mathrm{C}$ incubator with $5 \% \mathrm{CO}_{2}$.

Quantitative reverse transcription polymerase chain reaction ( $q R T-P C R)$. Total RNA was extracted from cells using TRIzol reagent (Thermo Fisher Scientific, Waltham, MA, USA) according to the manufacturer's instructions as previously described (8). Complementary DNA (cDNA) was synthesized from $5 \mu \mathrm{g}$ of total RNA with a random hexamer using the SuperScript III First-Strand synthesis system (Thermo Fisher Scientific). The cDNAs were used for the measurement of gene expression with a 7500 Real-time PCR system (Thermo Fisher Scientific) using TaqMan probes. The experiments were performed in triplicate with blinded patient information. Expression assays were purchased from Thermo Fisher Scientific (ANXA1, Hs00167549_m1) and $\beta$-actin was used as an internal control (Hs99999903_m1). Relative ANXA1 expression was calculated using the $2^{-\Delta \Delta C T}$ method, according to the supplier's protocol (Thermo Fisher Scientific).

Western blotting. Western blot analysis was performed as previously described (8). Cells were washed twice with ice-cold PBS and scraped. The cells were then centrifuged at $15,000 \mathrm{rpm}$ to pellet cellular debris and stored overnight at $-80^{\circ} \mathrm{C}$. The protein lysates were collected using ice-cold RIPA buffer containing Halt Protease Inhibitor single-use cocktail (Thermo Fisher Scientific). Protein concentration was determined by the Bradford method (Bio-Rad, Hercules, CA, USA). SDS-PAGE were prepared by mixing aliquots of the protein with Novex Tris-Glycine SDS sample buffer (Thermo Fisher Scientific) and heated at $100^{\circ} \mathrm{C}$ for $3 \mathrm{~min}$. The protein samples were run on $10 \%$ Bis-Tris gels at $100 \mathrm{~V}$ for $90 \mathrm{~min}$ with MES SDS running buffer (Thermo Fisher Scientific). For western blot analysis, gels were electro-transferred to a nitrocellulose membrane using the iBlot Dry Blotting system (Thermo
Fisher Scientific). Proteins were blocked using Starting Block (PBS) blocking buffer (Pierce, Rockford, IL, USA) and detected using anti-ANXA1 (clone 29), anti-hypoxia-inducible factor-1 $\alpha$ (HIF-1 $\alpha$ ) (BD Novus Bioscience Biologicals, Littleton, CO, USA), anti- $\beta$-actin (Santa Cruz Biotechnology, Dallas, TX, USA), and a goat anti-mouse secondary antibody phosphatase (Novagen, Billerica, MA, USA). Western blot analyses were then incubated with Super Signal West Pico detection system (Pierce) and detected using LAS-4000 IR MultiColor (Fujifilm, Tokyo, Japan).

Overexpression of ANXA1 expression using an expression vector. To generate the plasmid pcDNA3.1-ANXA1, ANXA1 was amplified by PCR using primers designed as follows: ANXA1-F, 5'-AGCTAGCACACTTTTTCAAAAATGGCAA TGG-3'; ANXA1-R, 5'-AGGATCCGGGAATGTTTAGTTTC CTCCACA-3'. These primers contained extragenic NheI and BamHI recognition sites (underlined), respectively. Total RNA was extracted by TRIzol reagent from the SW837 cell line, which highly expressed ANXA1, and $1 \mu \mathrm{g}$ RNA was reverse-transcribed into cDNA by the SuperScript III First-Strand Synthesis system. PCR was performed using Pfx50 DNA Polymerase (Thermo Fisher Scientific) to obtain a high fidelity PCR product. The reaction mix was initially heated to $94^{\circ} \mathrm{C}$ for $2 \mathrm{~min}$, and amplification was performed at $94^{\circ} \mathrm{C}$ for $15 \mathrm{sec}, 55^{\circ} \mathrm{C}$ for $30 \mathrm{sec}$, and $68^{\circ} \mathrm{C}$ for $90 \mathrm{sec}$, in 35 cycles, with a final 10 min extension at $72^{\circ} \mathrm{C}$. The PCR product was identified by $1 \%$ agarose gel electrophoresis then isolated by QIAquick Gel Extraction kit and purified by QIAquick PCR Purification kit (both from Qiagen, Valencia, CA, USA). Finally, the PCR product was digested with the restriction enzymes NheI and $B a m H I$, and inserted into the corresponding sites of the pcDNA3.1(+) vector (Thermo Fisher Scientific) using Ligation high (Toyobo, Tokyo, Japan).

One Shot TOP10 Competent Cells (Thermo Fisher Scientific) were used for transformation, then Qiagen Plasmid Midi kit (Qiagen) was used to isolate plasmids. One day before plasmid transfection, $5 \times 10^{5} \mathrm{RKO}$ cells were plated in $6-\mathrm{cm}$ plates. The cells were transfected with $1 \mu \mathrm{g}$ of plasmid DNA using Lipofectamine 2000 regent (Thermo Fisher Scientific) according to the manufacturer's instructions. After that, we selected a stable transfected cell line using the antibiotic $400 \mu \mathrm{g} / \mathrm{ml} \mathrm{G} 418$ (Roche, Indianapolis, IN, USA).

Knockdown of ANXAl expression using siRNA technology. The siRNA oligonucleotides for ANXA1 (HSS100502 and HSS100503) and the control were purchased from Thermo Fisher Scientific. The ANXA1 siRNA sequences were designed as follows: siRNA-1, 5'-CAACCAUCAUUGACAUUCUAAC UAA-3'; siRNA-2, 5'-GCCUUGCAUAAGGCCAUAAUGG UUA-3'. A scrambled siRNA was used as an internal control and $5 \times 10^{5}$ HCT 116 cells grown in 6-cm dishes were transfected with $40 \mathrm{nM}$ of each siRNA using Lipofectamine RNAiMAX (Thermo Fisher Scientific) according to the manufacturer's instructions.

Measurement of cell proliferation. The cell proliferation rate was assessed by cell counting kit-8 (CCK-8) (Dojindo, Tokyo, Japan), according to the manufacturer's instructions. Briefly, $1 \times 10^{4}$ cells of colon cancer cells, including control cells, were plated per well in 96-well plates and 5-FU was added. After 


\section{A $\quad$ sw480}

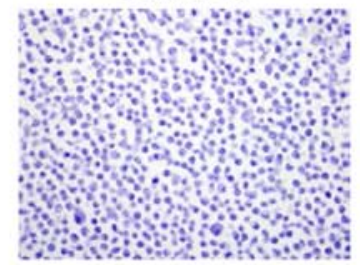

LS174T

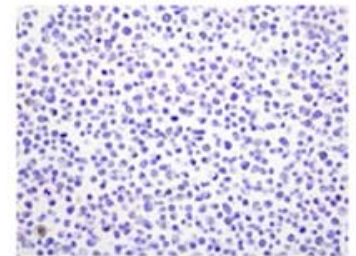

sW620

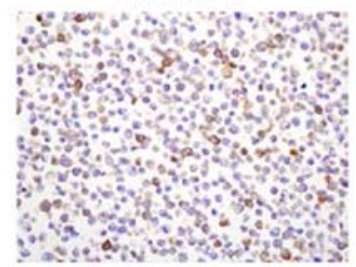

RKO

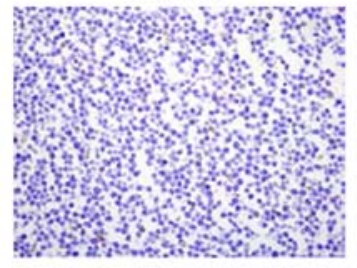

LoVo

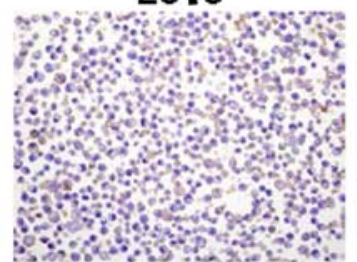

HCT116

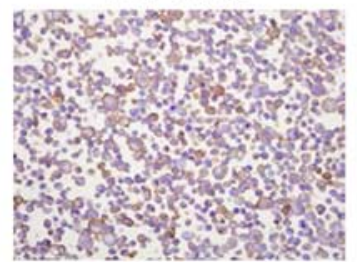

Colo201

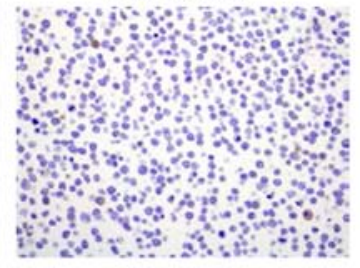

Colo205

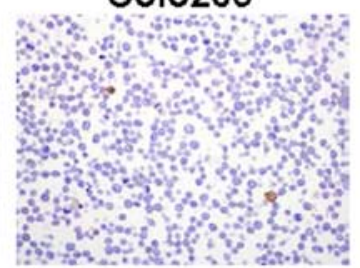

SW48

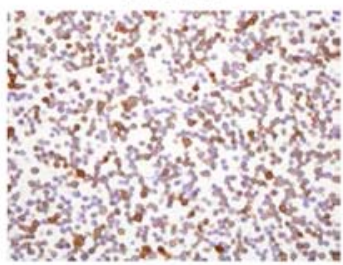

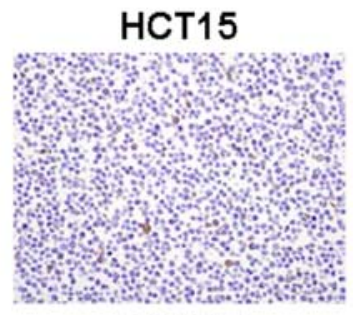

SW837

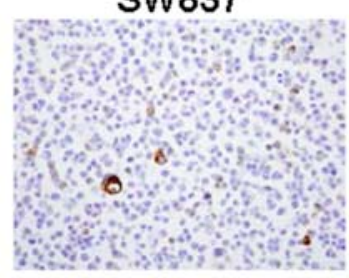

B

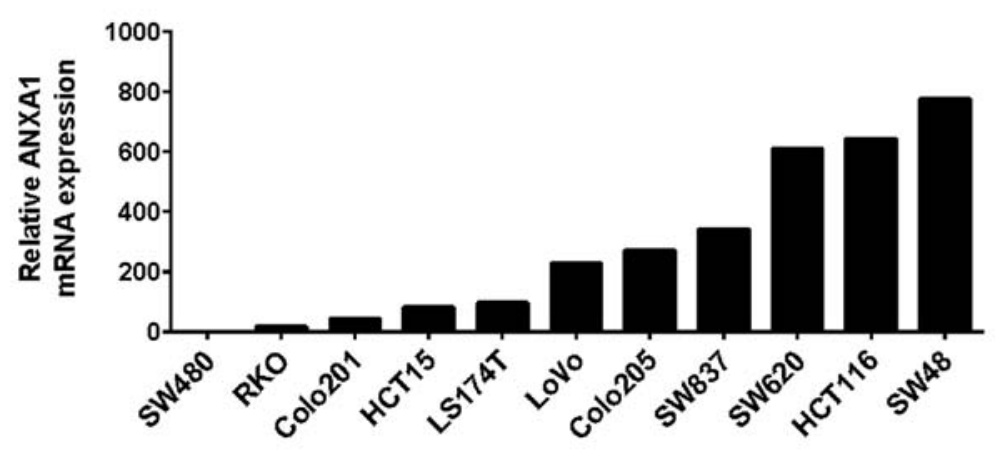

Figure 1. ANXA1 expression in colon cancer cell lines. (A) Immunohistochemical staining of ANXA1 in human colon cancer cell lines. Magnification, x400. (B) qRT-PCR analysis of ANXA1 expression in colon cancer cell lines. Relative ANXA1 mRNA expression levels to SW480 (SW480=1) are shown (normalized to $\beta$-actin).

$72 \mathrm{~h}, 10 \mu \mathrm{l}$ of CCK-8 reagent was added to each well. After $1 \mathrm{~h}$ of incubation at $37^{\circ} \mathrm{C}$, the absorbance was measured at $450 \mathrm{~nm}$ using a Benchmark Plus microplate reader (Bio-Rad).

Statistical data analysis. Statistical analysis was carried out with an unpaired Student's t-test using GraphPad Prism v5.0 (GraphPad Software Inc., La Jolla, CA, USA). P<0.05 was considered significant.

\section{Results}

ANXA1 expression in colon cancer cell lines. ANXA1 protein expression in ten human colon cancer cell lines (SW480, RKO, Colo201, HCT15, LS174T, LoVo, Colo205, SW620, HCT116 and SW48) were examined by IHC staining using an anti-ANXA1 antibody. Positive staining of ANXA1 was detected in the nucleus and cytoplasm of the SW620, HCT116 and SW48 cells, while negative staining of ANXA1 was found in the SW480, RKO, Colo201, HCT15 and LS174T cells (Fig. 1A).
To confirm ANXA1 expression by IHC evaluation in the cancer cell lines, we performed qRT-PCR using the same colon cancer cell lines. Consistent with the IHC results, the ANXA1 mRNA was highly expressed in the SW620, HCT116 and SW48 cells and significantly decreased in the SW480 and RKO cells, suggesting that ANXA1 expression was upregulated at the transcriptional level (Fig. 1B).

Overexpression of ANXA1 correlates to 5-FU resistance in colon cancer cells. To investigate the role of ANXA1 in 5-FU resistance, we constructed a 5-FU-resistant SW480 cell line (SW480/5-FU). First, we confirmed that there were no significant differences in cell proliferation between SW480 and SW480/5-FU cells (data not shown). To compare the growth inhibitory effect of 5-FU between the SW480 and SW480/5-FU cells, we then treated these cells with five different concentrations of 5-FU $(0,0.01,0.1,0.5$ and $1 \mu \mathrm{M})$ (Fig. 2A). The inhibitory concentration $50\left(\mathrm{IC}_{50}\right)$ indicated that the $5-\mathrm{FU}$ resistance level of the SW480/5-FU cells was 8-fold greater 

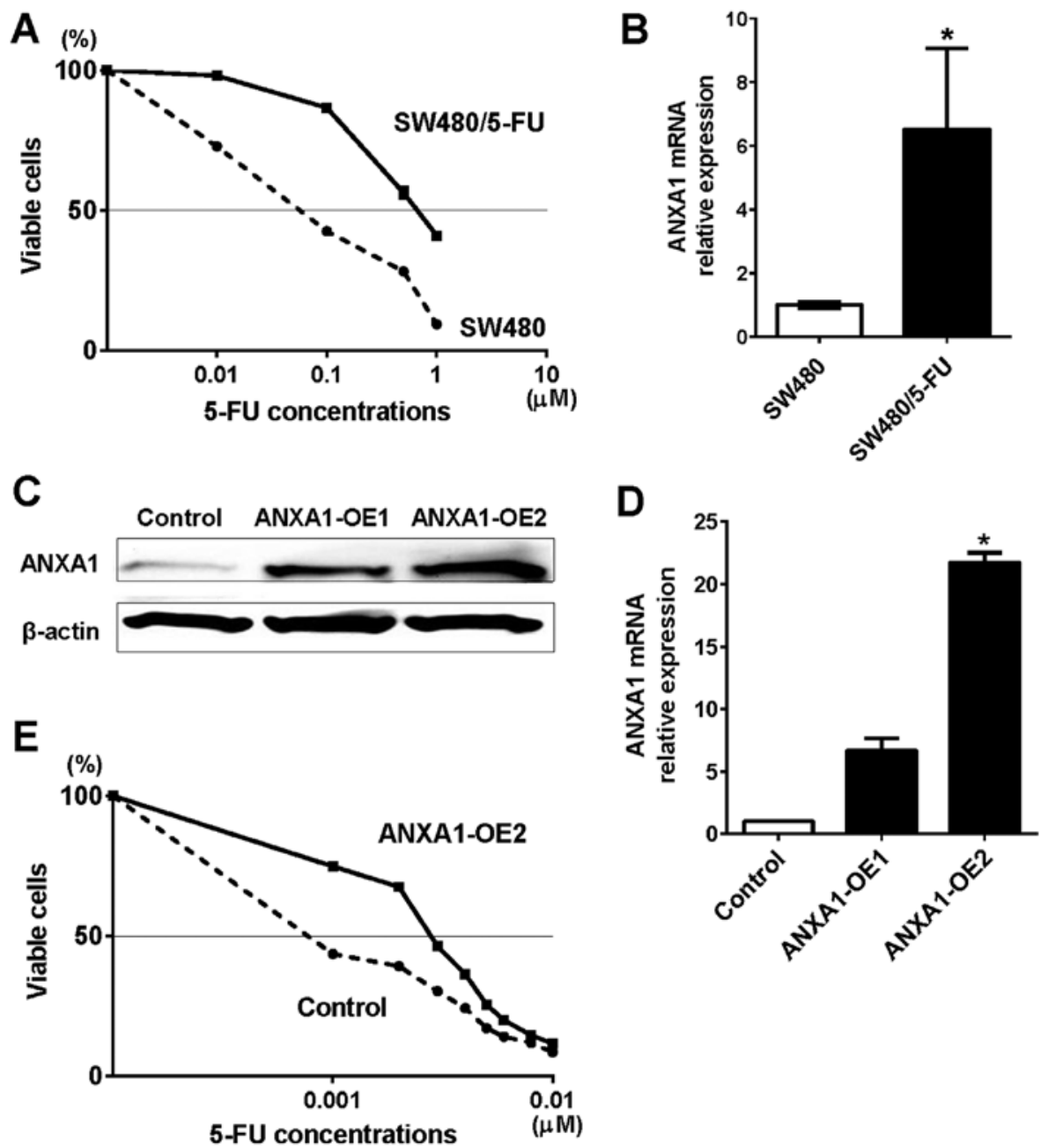

Figure 2. Increased cell viability against 5-FU in ANXA1 overexpression in colon cancer cells. (A) SW480/5-FU cells increased 5-FU resistance compared with control SW480 cells. Cell viability was measured by CCK-8 assay. (B) qRT-PCR analysis of ANXA1 in SW480/5-FU cells and control cells. Relative ANXA1 mRNA expression levels to control (control=1) are shown (normalized to $\beta$-actin). Data are mean \pm SD. "P<0.05. (C) Western blot analysis of ANXA1 overexpression in the RKO cells (ANXA1-OE1 and -OE2) and the control cells. $\beta$-actin was used as a loading control. (D) qRT-PCR analysis of ANXA1 overexpression in the RKO cells (ANXA1-OE1 and -OE2) and control cells. Relative ANXA1 mRNA expression levels are shown (normalized to $\beta$-actin). Data are mean $\pm \mathrm{SD} .{ }^{*} \mathrm{P}<0.05$. (E) ANXA1-OE2 cells increased 5-FU resistance compared with the control cells. Cell viability was measured by CCK-8 assay.

than that of the control cells. The mRNA expression of ANXA1 in SW480/5-FU cells was 6.5-fold higher than that of the control cells (Fig. 2B). These results suggest that 5-FU resistance induced ANXA1 expression in the colon cancer cells.

To examine whether ANXA1 overexpression affected 5-FU resistance, we overexpressed ANXA1 in the colon cancer cells and investigated cell proliferation. Both protein and mRNA expression of ANXA1 was upregulated by two independent clones (ANXA1-OE1 and -OE2) in RKO cells that originally expressed downregulated ANXA1 (Fig. 2C and D). While no morphological changes were observed in the ANXA1-OE2 cells, cell viability was attenuated (Fig. $2 \mathrm{E}$ ). The $\mathrm{IC}_{50}$ indicated that the 5-FU resistance level of the ANXA1-OE2 cells was 3 -fold greater than that of the control cells. These results further suggested that high ANXA1 expression is associated with 5-FU resistance in colon cancer cells.

Knockdown of ANXA1 correlates 5-FU sensitivity in colon cancer cells. According to our present and previous results, which indicated that ANXA1 expression was correlated with clinicopathological factors in colon cancer, we hypothesized that knockdown of ANXA1 improves 5-FU sensitivity. To verify this hypothesis, we used gene knockdown technology to confirm that both protein and mRNA ANXA1 expressions were downregulated by siRNA oligonucleotide in HCT116 cells (ANXA1-KD), which originally expressed upregulated ANXA1 (Fig. 3A and B). While no morphological changes were observed in the ANXA1-KD cells, cell viability was attenuated (Fig. 3C). The $\mathrm{IC}_{50}$ indicated that the 5-FU resistance level of the ANXA1-KD cells was 4-fold lower than that of the control cells. These results suggest that downregulation of ANXA1 significantly improved 5-FU sensitivity, reconfirming that ANXA1 is associated with 5-FU resistance in colon cancer cells. Of note, the growth inhibitory effect of 5-FU between SW480 and HCT116 cells was not significantly different (control cells in Figs. 2A and 3C). This result suggests that the spontaneous expression of ANXA1 affects 5-FU resistance only slightly in colon cells.

Induction of ANXA1 in hypoxia. To further assess the induction of ANXA1 during colon cancer progression, we examined 

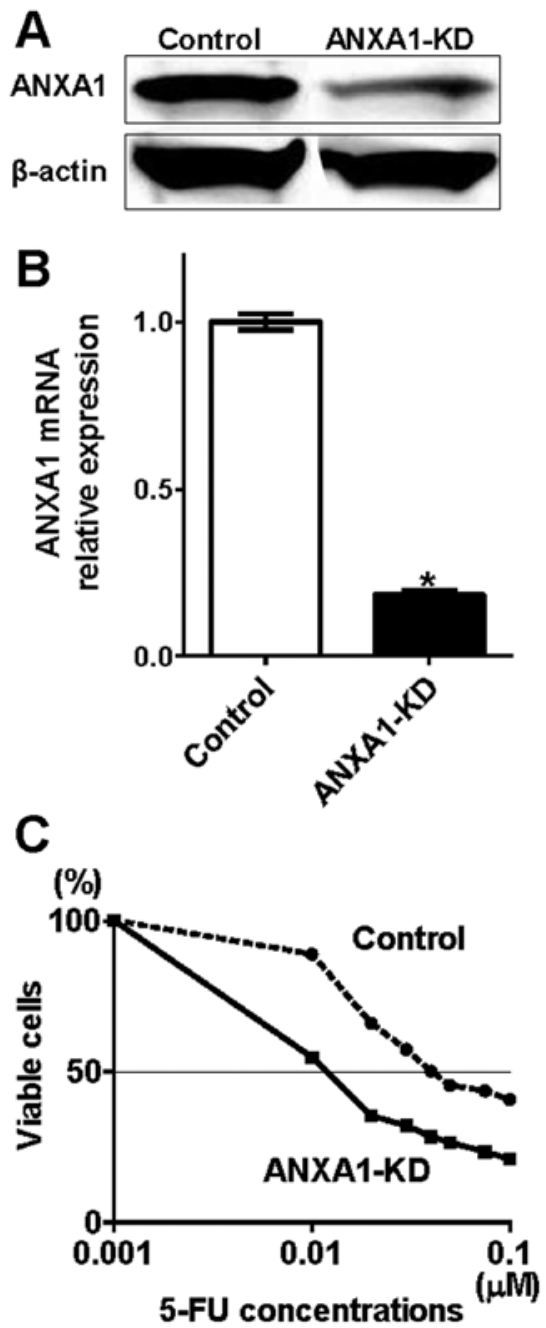

Figure 3. Reduced cell viability against 5-FU in ANXA1 knockdown in colon cancer cells. (A) Western blot analysis of ANXA1 knockdown in HCT116 cells (ANXA1-KD) and the control cells. $\beta$-actin was used as a loading control. (B) qRT-PCR analysis of ANXA1 knockdown in HCT116 cells (ANXA1-KD) and the control cells. Relative ANXA1 mRNA expression levels to control (control=1) are shown (normalized to $\beta$-actin). Data are mean \pm SD. ${ }^{*} \mathrm{P}<0.05$. (C) ANXA1-KD cells decreased 5-FU resistance compared with the control cells. Cell viability was measured by CCK- 8 assay.

ANXA1 expression under hypoxic conditions, is one of the main characteristic features of malignant tumors. Following treatment of the SW480 cells with a hypoxia mimic induced by $\mathrm{CoCl}_{2}$, ANXA1 and HIF-1 $\alpha$ expressions were evaluated. We observed that the HIF-1 $\alpha$ and ANXA1 proteins were induced by hypoxia (Fig. 4A). This induction of ANXA1 was also confirmed by examining mRNA expression (Fig. 4B). These results suggest that hypoxia may affect 5-FU resistance through induction of ANXA1 in colon cancer cells.

\section{Discussion}

In this study, we demonstrated that ANXA1 is associated with 5-FU resistance in colon cancer cells. We previously reported that upregulated ANXA1 was associated with cancer invasion and lymph node metastasis in colon cancer (9). Based on these findings, we further investigated the biological significance of ANXA1 by using overexpression and knockdown methods in colon cancer cells. Consistent with our findings, a recent
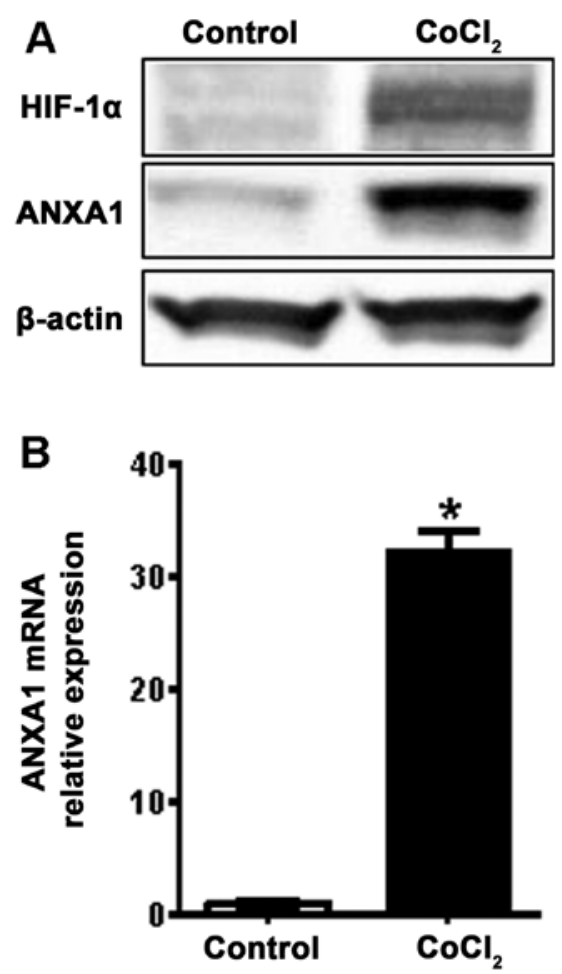

Figure 4. Induction of HIF-1 $\alpha$ and ANXA1 by hypoxia treatment. (A) Western blot analysis of hypoxia mimic induced by $\mathrm{CoCl}_{2}$ and the control in $\mathrm{SW} 480$ cells. $\beta$-actin was used as a loading control. (B) qRT-PCR analysis of ANXA1 expression with hypoxia and the control. Relative ANXA1 mRNA expression levels to control (control=1) are shown (normalized to $\beta$-actin). Data are mean $\pm \mathrm{SD} .{ }^{*} \mathrm{P}<0.05$

study has also revealed that upregulated ANXA1 was associated with resistance to various chemotherapeutic agents (10). ANXA1 activates NF- $\mathrm{kB}$, which is known as the association of resistance to 5-FU, resulting in progression of metastasis (12). The investigation of resistance mechanisms to chemotherapies is strongly required to develop anticancer therapies with high efficacy and minimum risk of adverse events. Although the exact mechanisms of ANXA1 in cancer remain unknown, it is worth investigating the role of ANXA1 in drug resistance.

This study implicated that overcoming 5-FU resistance by ANXA1 modulation may be of benefit for patients with colon cancer as well as other cancers. Because 5-FU is a key drug in both FOLFOX and FORFIRI regimens, which are the standard base regimens and used as a first- or second-line therapy for the treatment of colorectal cancer (13-16). Although 5-FU is one of the most investigated drugs in terms of anticancer activity, the molecular mechanism of resistance to 5-FU has yet to be fully elucidated (17). Both FOLFOX and FORFIRI regimens are highly effective; however, resistance to these therapies eventually occurs during treatment, resulting in tumor recurrence and metastasis. Therefore, the understanding of 5-FU resistance mechanism leads to the possibility to provide longer treatment effect and improve patient outcome when either FOLFOX or FORFIRI are used.

Drug resistance is also associated with hypoxia in malignant tumors (18). Hypoxia reduces chemotherapeutic effects by inhibiting tumor cell proliferation, inducing cell cycle arrest, and affecting various protein expressions (19). We have previously demonstrated that hypoxia and the hypoxia mimic 
induced by $\mathrm{CoCl}_{2}$ increased ANXA1 and HIF-1 $\alpha$ expression in breast cancer cells (8). HIF- $1 \alpha$ is a transcription factor that contains a basic helix-loop-helix motif as well as a PAS domain. HIF-1 $\alpha$ is induced by hypoxia and promotes tumor progression by interacting with TP53 (20-23). This study also revealed that HIF-1 $\alpha$ and ANXA1 were induced by hypoxia, re-suggesting a significant relationship between hypoxia and drug resistance by modulating HIF- $1 \alpha$ and ANXA1 expressions in colon cancer. However, the interaction between HIF- $1 \alpha$ and ANXA1 in colon cancer is currently unknown. Inhibition of HIF- $1 \alpha$ rescues multidrug resistance in colon cancer cells (24); therefore, inhibition of ANXA1 is also expected to overcome 5-FU resistance. Of note, one clinical trial (NCT00984048) to identify biomarkers in metastatic colorectal cancer patients that have acquired clinical resistance to first-line chemotherapy (FOLFOX/bevacizumab or FOLFIRI/bevacizumab) has started. This study allowed us to further understand drug resistance via a molecular signature.

In conclusion, we report that ANXA1 is upregulated in 5-FU-resistant colon cancer cells and ANXA1 inhibition can overcome 5-FU resistance. This suggests that modulating ANXA1 expression may provide an interesting strategy to overcome 5-FU resistance and provide benefits for colorectal cancer patients.

\section{Acknowledgements}

This study was supported by the JSPS KAKENHI, grant no. $15 \mathrm{k} 10143$.

\section{References}

1. Siegel RL, Miller KD and Jemal A: Cancer statistics, 2016. CA Cancer J Clin 66: 7-30, 2016.

2. Venook AP, Weiser MR and Tepper JE: Colorectal cancer: All hands on deck. Am Soc Clin Oncol Educ Book 34: 83-89, 2014.

3. Mayer RJ, Venook AP and Schilsky RL: Progress against GI cancer during the American Society of Clinical Oncology's first 50 years. J Clin Oncol 32: 1521-1530, 2014.

4. Rescher U and Gerke V: Annexins - unique membrane binding proteins with diverse functions. J Cell Sci 117: 2631-2639, 2004.

5. Lim LH and Pervaiz S: Annexin 1: The new face of an old molecule. FASEB J 21: 968-975, 2007.

6. Ydy LR, do Espírito Santo GF, de Menezes I, Martins MS Ignotti E and Damazo AS: Study of the Annexin A1 and its associations with carcinoembryonic antigen and mismatch repair proteins in colorectal cancer. J Gastrointest Cancer 47: 61-68, 2016.

7. Duncan R, Carpenter B, Main LC, Telfer C and Murray GI: Characterisation and protein expression profiling of annexins in colorectal cancer. Br J Cancer 98: 426-433, 2008.

8. Okano M, Kumamoto K, Saito M, Onozawa H, Saito K, Abe N, Ohtake T and Takenoshita S: Upregulated Annexin A1 promotes cellular invasion in triple-negative breast cancer. Oncol Rep 33 1064-1070, 2015.
9. Sato Y, Kumamoto K, Saito K, Okayama H, Hayase S, Kofunato Y, Miyamoto K, Nakamura I, Ohki S, Koyama Y, et al: Upregulated Annexin A1 expression in gastrointestinal cancer is associated with cancer invasion and lymph node metastasis. Exp Ther Med 2: 239-243, 2011.

10. Wang Y, Serfass L, Roy MO, Wong J, Bonneau AM and Georges E: Annexin-I expression modulates drug resistance in tumor cells. Biochem Biophys Res Commun 314: 565-570, 2004.

11. Takahashi K, Tanaka M, Inagaki A, Wanibuchi H, Izumi Y, Miura K, Nagayama K, Shiota M and Iwao H: Establishment of a 5-fluorouracil-resistant triple-negative breast cancer cell line. Int J Oncol 43: 1985-1991, 2013.

12. Anbalagan D, Yap G, Yuan Y, Pandey VK, Lau WH, Arora S, Bist P, Wong JS, Sethi G, Nissom PM, et al: Annexin-A1 regulates microRNA-26b* and microRNA-562 to directly target $\mathrm{NF}-\kappa \mathrm{B}$ and angiogenesis in breast cancer cells. PLoS One 9: e114507, 2014.

13. de Gramont A, Figer A, Seymour M, Homerin M, Hmissi A, Cassidy J, Boni C, Cortes-Funes H, Cervantes A, Freyer G, et al: Leucovorin and fluorouracil with or without oxaliplatin as firstline treatment in advanced colorectal cancer. J Clin Oncol 18: 2938-2947, 2000.

14. Goldberg RM, Sargent DJ, Morton RF, Fuchs CS, Ramanathan RK, Williamson SK, Findlay BP, Pitot HC and Alberts SR: A randomized controlled trial of fluorouracil plus leucovorin, irinotecan, and oxaliplatin combinations in patients with previously untreated metastatic colorectal cancer. J Clin Oncol 22: 23-30, 2004.

15. Douillard JY, Cunningham D, Roth AD, Navarro M, James RD, Karasek P, Jandik P, Iveson T, Carmichael J, Alakl M, et al: Irinotecan combined with fluorouracil compared with fluorouracil alone as first-line treatment for metastatic colorectal cancer: A multicentre randomised trial. Lancet 355: 1041-1047, 2000.

16. Tournigand C, André T, Achille E, Lledo G, Flesh M, Mery-Mignard D, Quinaux E, Couteau C, Buyse M, Ganem G, et al: FOLFIRI followed by FOLFOX6 or the reverse sequence in advanced colorectal cancer: A randomized GERCOR study. J Clin Oncol 22: 229-237, 2004.

17. Longley DB, Harkin DP and Johnston PG: 5-Fluorouracil: Mechanisms of action and clinical strategies. Nat Rev Cancer 3: 330-338, 2003.

18. Gottesman MM: Mechanisms of cancer drug resistance. Annu Rev Med 53: 615-627, 2002.

19. Gardner LB, Li Q, Park MS, Flanagan WM, Semenza GL and Dang CV: Hypoxia inhibits G1/S transition through regulation of p27 expression. J Biol Chem 276: 7919-7926, 2001.

20. Giatromanolaki A, Koukourakis MI, Sivridis E, Turley H, Talks K, Pezzella F, Gatter KC and Harris AL: Relation of hypoxia inducible factor 1 alpha and 2 alpha in operable non-small cell lung cancer to angiogenic/molecular profile of tumours and survival. Br J Cancer 85: 881-890, 2001.

21. Liu L, Ning X, Sun L, Zhang H, Shi Y, Guo C, Han S, Liu J, Sun S, Han Z, et al: Hypoxia-inducible factor-1 alpha contributes to hypoxia-induced chemoresistance in gastric cancer. Cancer Sci 99: 121-128, 2008.

22. An WG, Kanekal M, Simon MC, Maltepe E, Blagosklonny MV and Neckers LM: Stabilization of wild-type p53 by hypoxia-inducible factor 1alpha. Nature 392: 405-408, 1998.

23. Ravi R, Mookerjee B, Bhujwalla ZM, Sutter CH, Artemov D, Zeng Q, Dillehay LE, Madan A, Semenza GL and Bedi A: Regulation of tumor angiogenesis by p53-induced degradation of hypoxia-inducible factor 1alpha. Genes Dev 14: 34-44, 2000.

24. Chen J, Ding Z, Peng Y, Pan F, Li J, Zou L, Zhang Y and Liang H: HIF-1 $\alpha$ inhibition reverses multidrug resistance in colon cancer cells via downregulation of MDR1/P-glycoprotein. PLoS One 9: e98882, 2014. 